\title{
Development of electrochemical methods to enzymatically detect traumatic brain injury biomarkers
}

Brittney A. Haselwood ${ }^{\mathrm{a}}$, MS, Jeffrey T. La Belle ${ }^{\mathrm{a}, \mathrm{b}}$, Ph.D.

${ }^{a}$ School of Biological and Health Systems Engineering, Arizona State University, 550 E. Orange St., Tempe, AZ 85287, USA.

${ }^{\mathrm{b}}$ School of Medicine, Mayo Clinic Arizona, 13400 E. Shea Blvd., Scottsdale, AZ 85259, USA.

Corresponding author: Jeffrey T. La Belle, Ph.D. Tel. +1 480-727-9061. Fax +1 480-727-7624.

Email: JEFFREY.LABELLE@ asu.edu. Postal address: School of Biological and Health Systems Engineering, Arizona State University, 501 E Tyler Mall, Tempe, AZ 85287-9709, USA.

\begin{abstract}
As awareness of traumatic brain injury (TBI) increases, the need to detect mild forms and distinguish between the different severities of TBI becomes more apparent. The goal of this work is to develop a point-of-care sensor to detect whole blood biomarkers for rapid and sensitive diagnosis of TBI severity. Presented herein is the enzymatic detection of norepinephrine through the use of immobilization chemistry and impedance techniques. Sustained elevation of norepinephrine concentrations in the blood has been correlated to negative long-term outcomes in TBI cases, often resulting in permanent cognitive or physical deficits.
\end{abstract}

Novel analysis techniques have been used to identify an optimal binding frequency $(371.1 \mathrm{~Hz})$ of norepinephrine to the immobilized enzyme on a gold disk electrode. This form of analysis yielded a logarithmic fit characterized by exceptional responsivity $\left(20.89 \mathrm{ohms} / \mathrm{pgmL}^{-1}\right)$, reproducibility $\left(R^{2}=0.96\right)$, and lower limit of detection $(98 \mathrm{pg} / \mathrm{mL})$ first in purified samples, then in rabbit whole blood solutions. Once the optimal binding frequency was determined, the preliminary use of an impedance-time technique was attempted in this work. This technique more closely resembles the amperometric detection method used in commercial self-monitoring blood glucose meters, allowing for continuous or instantaneous measurement of blood borne biomarkers without compromising sensitivity. Future directions include exploration of simultaneous multi-marker detection with the impedance-time technique and experimentation with novel mesoporous materials to filter large blood components.

\section{Keywords}

Norepinephrine; Traumatic Brain Injury; Impedance; Point-of-Care Technology; Electrochemistry; Enzyme

\section{Introduction}

Each year 1.4 million and 1 million people in the United States and Europe, respectively, visit hospitals and urgent care centers to be treated for traumatic brain injury (TBI) (Brain Injury 
Facts, 2011; Faul et al., 2010). In 2010, the estimated costs associated with TBI were $\$ 76.5$ billion, averaging $\$ 45,000$ per injury in the US (Finkelstein et al., 2006). Unfortunately, TBIs are easy to miss as $75 \%$ of TBIs are mild with little to no apparent symptoms, while multiple mild TBIs occurring in quick succession can cause life-altering brain damage or death (National Center for Injury Prevention and Control, 2003). Moreover, in mild and moderate injury cases, the brain will continue to sustain secondary damage during the extensive healing process which can be prolonged without treatment (National Hospital Discharge Survey, 2011).

Today, several devices and methods exist to detect TBI. The most traditional method used clinically is the Glasgow Coma Scale (GCS) based on verbal, eye and motion responses which is often augmented with computer tomography imaging in moderate to severe cases (Svetlov et al., 2010; Hergenroeder et al., 2008). Current devices to monitor head trauma include piezoelectric or acceleration sensors that line a helmet for military or contact sport use (Daniel et al., 2010; Greenwald et al., 2008). Other non-commercial devices are biosensors which measure TBI biomarkers in cerebral spinal fluid or blood (Svetlov et al., 2010). These biosensors are particularly useful in quantitatively diagnosing TBI severity, whereas the piezoelectric sensors do not yield any direct physiological information (Chamoun et al., 2009). However, as advantageous as these detection platforms may be, they are not yet portable nor are they continuous in physiological state detection (which could be advantageous in intensive care cases).

Literature has shown that the catecholamines, including norepinephrine, have a positive correlation with trauma such as brain injury (Clifton et al., 1981; Mautes et al., 2001; Salim et al., 2009). Generally, asymptomatic levels of the catecholamines are $0 \mathrm{nM}$ to $10 \mathrm{nM}(0-1750$ $\mathrm{pg} / \mathrm{mL}$ ) in urine and $0.19 \mathrm{nM}$ to $11 \mathrm{nM}(33.25-1925 \mathrm{pg} / \mathrm{mL})$ in blood (Kumar et al., 2011; Mayo Clinic Laboratories, 2012). As a result of trauma, catecholamine blood concentrations fluctuate and can reach levels of $29 \mathrm{nM}(49 \mathrm{ng} / \mathrm{mL}$ ) or higher, depending on severity (Hamill et al., 1987). However, the current method to measure catecholamine levels is conducted in clinical laboratories by high-performance liquid chromatography (HPLC) on blood or urine samples (Mayo Clinic Laboratories, 2012; Tsunoda et al., 2009). Other developed non-clinical methods of catecholamine detection include electrochemical techniques in conjunction with various nanosensors or coatings, and purification methods such as hydrophilic interaction chromatography (Atta et al., 2010; Kim et al., 2010; Kumar et al., 2011; Prasad et al., 2009). Disadvantages of these methods include the need for a clinical chemistry laboratory to conduct the assay, time and resource costs for sample transport, stabilizing reagents and purification steps (Atta et al., 2010; Kumar et al., 2011; Mayo Clinic Laboratories, 2012; Prasad et al., 2009). To be useful in situations where the symptoms are transient, catecholamine levels would need to be measured frequently and rapidly, even at the bedside, which current assay methods do not permit. Despite these drawbacks, these measurement methods have achieved sufficient lower limits of detection (LLD) to reach the physiological levels of catecholamines. 
A promising electrochemical technique, often called the impedance-time technique, has been developed and used for a variety of purposes including monitoring cell proliferation on a surface and sensing DNA hybridization within 5-10 nM concentrations (Ghindilis et al., 2009; Upadhyay et al., 2000). The continuous-time and high sensitivity aspects of this technique bears striking similarities to the glucose meter's amperometric technique which shows promise as a TBI pointof-care diagnostic. As our understanding of TBI improves and as public and government concern regarding TBI rises in light of contact sport injuries and modern combat deaths, the need for a point-of-care technology to rapidly diagnose TBI has never been greater. To address this gap, this work explores impedance-based electrochemical techniques which have shown promise as point-of-care modalities with the sensitivity and specificity required to diagnose TBI in complex media.

\section{Material and Methods}

2.1 Chemical Reagents

All chemical reagents were purchased form Sigma-Aldrich, St. Louis, MO, USA unless otherwise stated. Potassium hexacyanoferrate (III) was dissolved in $10 \mathrm{mM}$ Phosphate Buffer Saline (EMD Chemicals, Billerica, MA, USA), henceforth called redox probe. Phenylethanolamine N-methyl transferase (PNMT) used in the electrochemical studies to detect Norepinephrine was purchased from Calzyme (Tulelake, CA, USA) and immobilized using 1 $\mathrm{mM}$ 16-mercaptohexadecanoic acid (16-MHDA) in ethanol, $10 \mathrm{mM}$ N-(3Dimethylaminopropyl)-N'-ethylcarbodiimide (in PBS, henceforth called EDC), 80mM (in PBS) N-Hydroxysulfosuccinimide Sodium Salt, henceforth called NHS (Toronto Research Chemicals, Toronto, Ontario, Canada), and 1\% (in DI) Ethanolamine. The DL-Norepinephrine Hydrochloride solutions with excess amounts of the PNMT co-factor, S-(5'-Adenosyl)-Lmethionine chloride dihydrochloride (SAM) were made into various concentrations ranging from $5.9 \mathrm{pM}$ to $59.1 \mathrm{nM}(1-10,000 \mathrm{pg} / \mathrm{mL})$ of $\mathrm{NE}$ in $10 \mathrm{mM}$ PBS solution at $\mathrm{pH}$ 7.4. White New Zealand rabbit whole blood stabilized with K2-EDTA was purchased from Bioreclamation LLC (New York, USA).

\subsection{Electrochemical Cell}

The electrochemical cell included a $2 \mathrm{~mm}$ gold disk working electrode (GDE), a Ag/AgCl wire reference electrode, and a Platinum wire counter electrode (purchased form $\mathrm{CH}$ Instruments, Austin, TX, USA). The electrochemical cell was established by placing a trimmed $1 \mathrm{~mL}$ pipette tip onto the GDE and bending the $\mathrm{Ag} / \mathrm{AgCl}$ electrode $(\mathrm{d}=0.5 \mathrm{~mm})$ and $\mathrm{Pt}$ electrode $(\mathrm{d}=0.5 \mathrm{~mm})$ wires into the cut pipette tip. Alligator clips connected the cell to the $660 \mathrm{C} \mathrm{CH}$ Instruments Electrochemical Analyzer. The samples pipetted into the well were a mixture of PBS and/or whole rabbit blood, norepinephrine, and $100 \mathrm{mM}$ ferricyanide redox probe. Prior to functionalization, cyclic voltammetry (6 segments, 1 to $-1 \mathrm{~V}$ ) was performed on clean GDEs 
using $100 \mu \mathrm{L}$ sample of redox probe to determine the formal potential (average of oxidation and reduction peak voltages).

\subsection{Functionalization}

The process of preparing the GDE can be found in La Belle et al., 2013 and is illustrated in scheme 1 , but is briefly described here: the GDE is cleaned using $\mathrm{Al}_{2} \mathrm{O}_{3}$ polishing pads and sonicated in DI water for 20 mins, then the alkane-thiol self-assembling monolayer (1 mM 16MHDA dissolved in ethanol) is incubated on the GDE surface for $1 \mathrm{hr}$. Next, a $10 \mathrm{mM}$ EDC/80 $\mathrm{mM}$ NHS in PBS solution is placed on the GDE surface for $1 \mathrm{hr}$ to prepare the carboxyl end of the 16-MHDA for protein attachment. Then, the enzyme $(0.01 \mathrm{mg}$ of PNMT in $1 \mathrm{~mL}$ PBS) is incubated on the modified GDE surface for $1 \mathrm{hr}$. Finally, $1 \%$ ethanolamine in DI is incubated on the sensor surface for 30 mins to prevent non-specific binding on any remaining bare gold and unbound 16-MHDA. After functionalization, the GDEs were stored in PBS at $4{ }^{\circ} \mathrm{C}$ until the $\mathrm{NE}+\mathrm{SAM}$ concentration gradients were run.

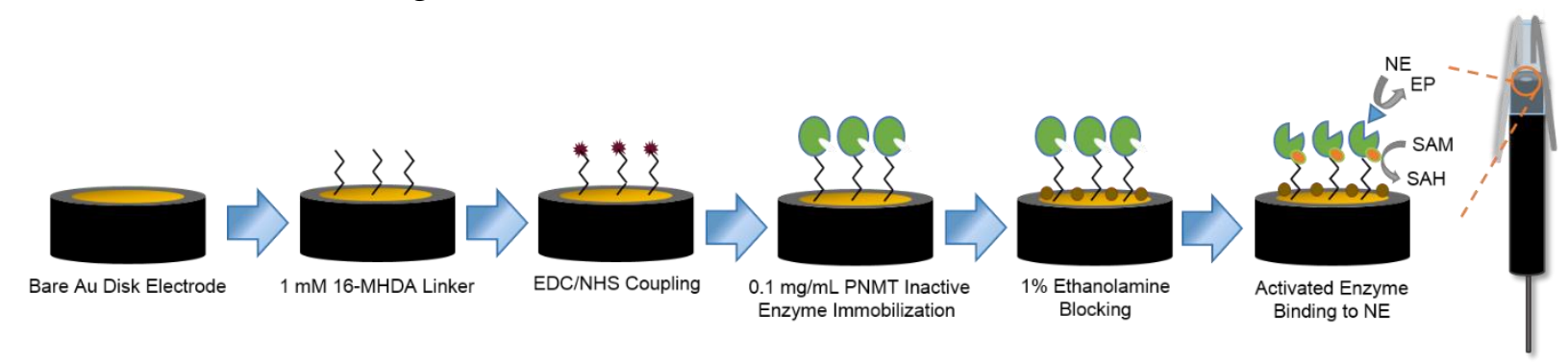

\section{Scheme 1.}

A schematic illustrating the immobilization chemistry process to functionalize the PNMT enzyme onto a GDE. Once functionalized, a solution containing SAM will activate the enzyme and allow NE binding to result in current production and impedance changes.

\subsection{Electrochemical Impedance Spectroscopy (EIS)}

The EIS method uses an AC voltage signal which changes frequency to stimulate the solution while current is being measured. Based on this measured current, impedance and phase are calculated in real-time, then plotted in the Nyquist graphs as shown in Fig1a. To achieve this, the following parameters were set: Initial $\mathrm{E}=$ formal potential found in the $\mathrm{CV}$ prior to functionalization, High Frequency $=100 \mathrm{kHz}$, Low Frequency $=1 \mathrm{~Hz}$, and Amplitude $=5 \mathrm{mV}$. After the GDE is functionalized, a NE+SAM in PBS serial dilution gradient consisting of 10 solutions ranging from 1-10,000 pg/mL (or $5.9 \mathrm{pM}-59.1 \mathrm{nM}$ of $\mathrm{NE}$ ) is run from lowest to highest concentration. These solutions encompass the entire physiological range and include an excess amount of SAM co-factor in PBS. Each solution was $50 \mu \mathrm{L}$ of NE+SAM in PBS mixture and $50 \mu \mathrm{L}$ of $100 \mathrm{mM}$ ferricyanide redox probe.

For the rabbit whole blood experiments, the solutions had the same effective concentration of NE and SAM but were mixed into 5\%,10\%, 25\%, and 90\% whole blood in PBS solutions. For example, the $10 \%$ whole blood solution tested was $25 \mu \mathrm{L}$ of $200 \mathrm{mM}$ ferricyanide, $25 \mu \mathrm{L}$ of $4 \mathrm{x}$ 
NE+SAM in PBS solution used in the purified case, $10 \mu \mathrm{L}$ of whole blood in $40 \mu \mathrm{L}$ of PBS. The $90 \%$ whole blood solution was $5 \mu \mathrm{L}$ of $1000 \mathrm{mM}$ ferricyanide, $5 \mu \mathrm{L}$ of $20 \mathrm{x} \mathrm{NE}+\mathrm{SAM}$ in PBS solution used in the purified case, and $90 \mu \mathrm{L}$ of whole blood. This data was obtained the same way as the purified data: a blank of only PBS and redox probe was first run, followed by the NE concentration gradient.

\subsection{Impedance-Time (Z-t)}

The Impedance-Time technique is similar to the EIS method. Instead of sweeping over a frequency range, only one frequency is applied over time. The resulting data is plotted as the complex impedance versus time as shown in Figs 2a and 2c. In the Z-t experiments, the following parameters were set: Initial $\mathrm{E}=$ formal potential found in the $\mathrm{CV}$ prior to functionalization, Amplitude $=5 \mathrm{mV}$, Frequency= any frequency of interest in $\mathrm{Hz}$, Sample Interval $=1 \mathrm{sec}$, Run Time= any time range in sec. The first set of Z-t experiments were run on the purified 1-10,000 $\mathrm{pg} / \mathrm{mL} \mathrm{NE}+\mathrm{SAM}$ concentration gradient for $90 \mathrm{sec}$ at $368 \mathrm{~Hz}$. The second set of Z-t experiments were continuous impedance measurements as the initial sample of $50 \mu \mathrm{L}$ of $100 \mathrm{mM}$ ferricyanide was spiked with $500 \mathrm{pg} / \mathrm{mL} \mathrm{NE}+\mathrm{SAM}$ in PBS at 30, 40, 85, 145, and $325 \mathrm{sec}$ time points after initialization of the Z-t assay.

\section{Results and Discussion}

\subsection{EIS Results}

Fig. 1a overlays the Nyquist curves for the blank $(0 \mathrm{pg} / \mathrm{mL}), 1 \mathrm{pg} / \mathrm{mL}$ and $5,000 \mathrm{pg} / \mathrm{mL}$ $\mathrm{NE}+\mathrm{SAM}$ solutions run using EIS (though all 10 solutions were run). The black curves indicate the purified NE+SAM solutions made using only PBS and the redox probe. The gray curves refer to the $10 \%$ whole blood data collected for the same effective NE+SAM concentrations. These data were fitted to the classical Randles Equivalent Circuit R(CR) model and found to have a $\chi^{2}$ ranging from $1.01 \mathrm{E}-02$ to $1.42 \mathrm{E}-02$ in the purified case and $3.94 \mathrm{E}-02$ to $4.86 \mathrm{E}-02$ in the $10 \%$ whole blood case, indicating that the impedance Nyquist curves closely resemble the Randles Model. The components of this Randles model include a solution resistance, an electron transfer resistance, and a double layer capacitor caused by the SAM which separates changes from the GDE. It is evident from these Nyquist curves that the blood solution yields a greater responsivity than the purified solutions. This is likely due to the larger proteins such as albumin a blood cells aggregating near the electrode surface. However, this does not prevent specific binding of NE to the PNMT enzyme as the impedance increases in a logarithmic fashion as a function of NE concentration. Fig. 1b shows the calibration curves for each solution case (pure and $10 \%$ whole blood) at the determined optimal frequency of $371.1 \mathrm{~Hz}$. Ideally, a hand-held meter could be programed with these equations, and after running EIS on an unknown sample, this calibration curve could be used to convert the measured impedance into a concentration of $\mathrm{NE}$ in a complex media. Fig. 1c illustrates the challenge of selecting an optimal frequency. It is evident that the relationship between NE concentration and complex impedance is logarithmic, 
but there is a tradeoff between responsivity (slope) and reproducibility $\left(\mathrm{R}^{2}\right)$ for these logarithmic fits. Fig. 1c shows an $n=1$ purified case, but after several PNMT immobilizations and NE+SAM gradients $(\mathrm{N}=12), 371.1 \mathrm{~Hz}$ was chosen as it consistently had an $\mathrm{R}^{2}>0.95$, comparatively consistent slopes around $20 \mathrm{ohms} / \mathrm{pgmL}^{-1}$, from which an LLD of $98 \mathrm{pg} / \mathrm{mL}(579 \mathrm{pM})$ of NE was determined. For the clinical standard HPLC detection mechanism, the lower ranges of detection are $0.5-2.5 \mathrm{ng} / \mathrm{mL}$, whereas the ELISA method can detect concentrations of $10 \mathrm{pg} / \mathrm{mL}$, while another novel modified electrode using a differential pulse voltammetry could detect catecholamines in the lower $\mu \mathrm{M}(80+\mathrm{ng} / \mathrm{mL})$ range (Atta et al., 2010; Raggi et al., 1999; Svetlov et al., 2010). However, these techniques may require filtering of samples (whereas the whole blood samples in this publication are merely diluted), require many hours of assay time, or requires time-consuming data analysis. Fig. 1d confirms the issue of complex media causing noise in the impedance signal at the optimal frequency of $371.1 \mathrm{~Hz}$. The 5, 10 and $25 \%$ blood solutions seem to follow a logarithmic pattern, but the $90 \%$ whole blood solution becomes less predictable. However, this still shoes promise as a point-of-care technology. In a test strip form factor, the strip could contain some amount of PBS which will dilute the whole blood to an optimal percentage. This is feasible since head injury increases norepinephrine blood levels, the LLD should be sufficient to diagnose a head injury.

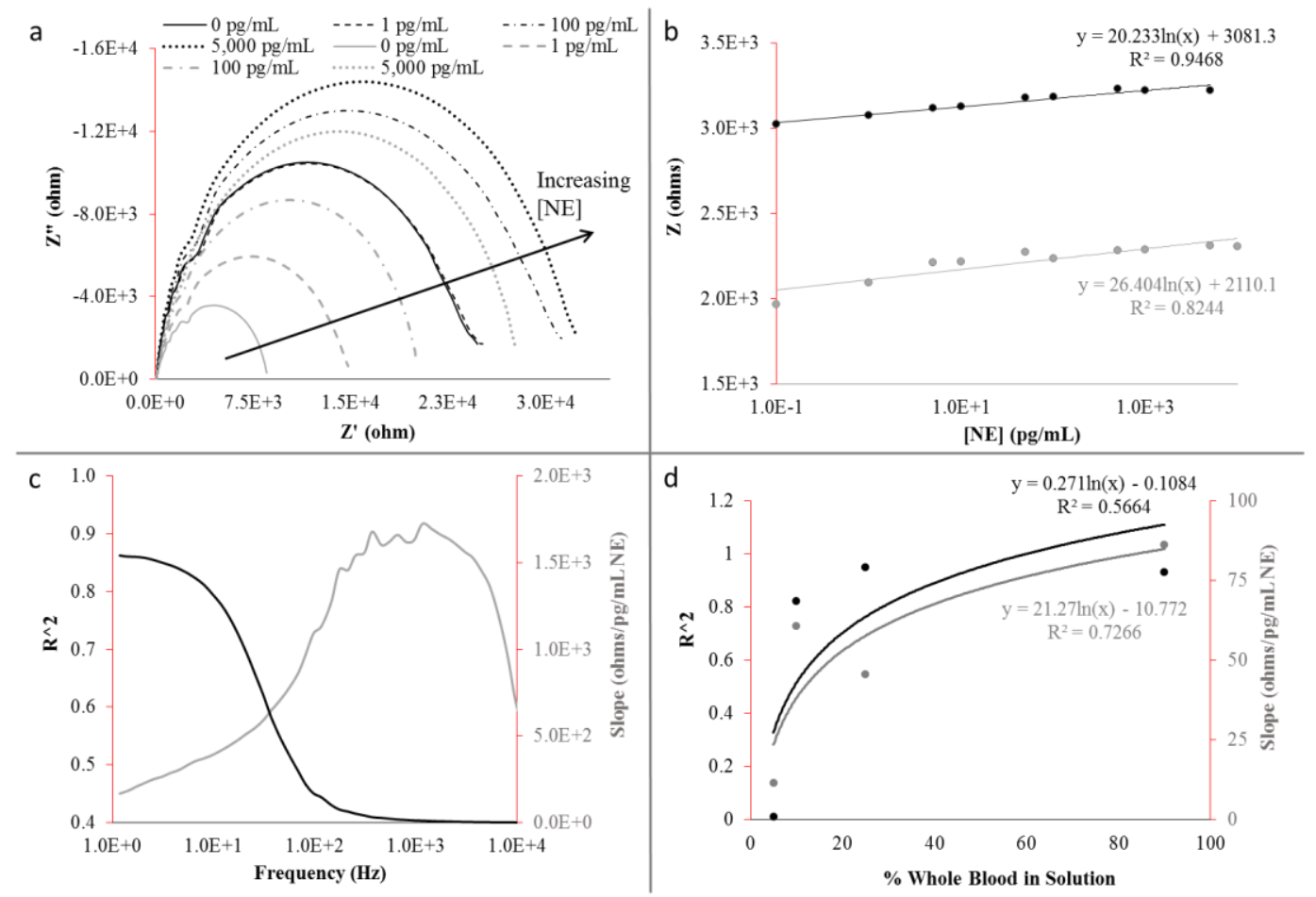

Fig. 1.

(a) Overlay of Nyquist curves resulting from impedance readings on 2 PNMT immobilized GDEs in purified (black) and $10 \%$ whole blood (gray) NE+SAM solutions. (b) Calibration curves plotting impedance as a function of NE concentration at the $371.1 \mathrm{~Hz}$ optimal binding frequency in purified (black) and $10 \%$ whole blood (gray) solutions. (c) This graph shows the relationship between a logarithmic fit $\mathrm{R}^{2}$ versus a logarithmic fit slope. In the case of 
PNMT and NE, there is a tradeoff between reproducibility and responsivity. (d) This is a comparison of logarithmic fit $\mathrm{R}^{2}$ (black) versus a logarithmic fit slope (gray) at $371.1 \mathrm{~Hz}$ as a function of $\%$ whole blood in the NE+SAM solution.

\subsection{Z-t Results}

The Z-t technique was attempted in an effort to reduce the required amount of hardware which would need to be miniaturized for a hand-held point-of-care technology. Additionally, this technique can be used to more thoroughly investigate and optimize the frequency selected for stimulation. Z-t applies only one frequency instead of several as in EIS, this can certainly be achieved without changing the detection mechanism or compromising sensitivity.

Fig. 2a corresponds to the first set of data collected using Z-t. Each NE+SAM solution (1-10,000 $\mathrm{pg} / \mathrm{mL}$ ) was run for $90 \mathrm{sec}$, the same amount of time for an EIS run. Both sets of data (black and gray) are similar GDEs experiencing the same solutions. However, there is a basal shift between the two theoretically same GDEs, and a slight basal shift as the concentration of NE increases. This is likely to be a temperature effect, observable after many consecutive runs. The total time of this data collection was over 45 minutes at room temperature, which is impressive from a stability standpoint. Additionally, the sensitivity increases as the resulting LLD $=8 \mathrm{pg} / \mathrm{mL}$ (47.29 $\mathrm{pM}$ ). This preliminary Z-t LLD is based on $\mathrm{N}=2$ purified data while the EIS LLD is based on $\mathrm{N}=12$ purified and whole blood data, but it shows promise for being as sensitive as a sandwich ELISA with a sensitivity of $10 \mathrm{pg} / \mathrm{mL}$ reported by Svetlov, et al (2010). However the Z-t method requires significantly less assay time; if the electrodes are prepared, quantification of a biomarker can be done in seconds.

Fig. $2 \mathrm{~b}$ shows the process of selecting an optimal time to collect the impedance data. According to these data, $3 \mathrm{sec}$ after assay initialization provides a strong correlation to NE concentration. This is a key finding if a hand-held meter can be programmed to stimulate the solution with a $371.1 \mathrm{~Hz}$ frequency, the solid black calibration equation could be used to convert impedance to NE concentration based on only $3 \mathrm{sec}$ of data, rivaling commercial glucose meters.

The last set of data collected with the Z-t technique explored different frequencies using 500 $\mathrm{pg} / \mathrm{mL}$ purified NE+SAM solutions for optimization purposes. Fig. 2c shows continuous spiking at the same intervals of PBS or $500 \mathrm{pg} / \mathrm{mL} \mathrm{NE}+\mathrm{SAM}$ solutions. There is an observable difference between the two applied frequencies, which is more significant than a small basal impedance difference. This solidifies our EIS findings that there is an optimal frequency at which analyte-protein binding occurs. As shown in Fig. 2 , the average $(\mathrm{N}=2)$ peak height corresponding to NE injections are more consistent at the $368 \mathrm{~Hz}$ frequency as opposed to the $321 \mathrm{~Hz}$ frequency. Two other frequencies, 3676 and $46300 \mathrm{~Hz}$, were also tested (data not shown) to determine if a harmonic relationship existed and to try a "dead" frequency. The $3676 \mathrm{~Hz}$ harmonic data showed no response to $500 \mathrm{pg} / \mathrm{mL}(2.96 \mathrm{nM}) \mathrm{NE}+\mathrm{SAM}$ solutions. The same was observed with the "dead" $46300 \mathrm{~Hz}$ response, as expected since biologically relevant binding 
frequencies lie below $10 \mathrm{kHz}$ (Adamson et al., 2012; Fairchild et al., 2009; Gonzales and La Belle, 2012; La Belle et al., 2007). A high "dead" frequency however, may become important for calibration purposes in a future point-of-care technology.

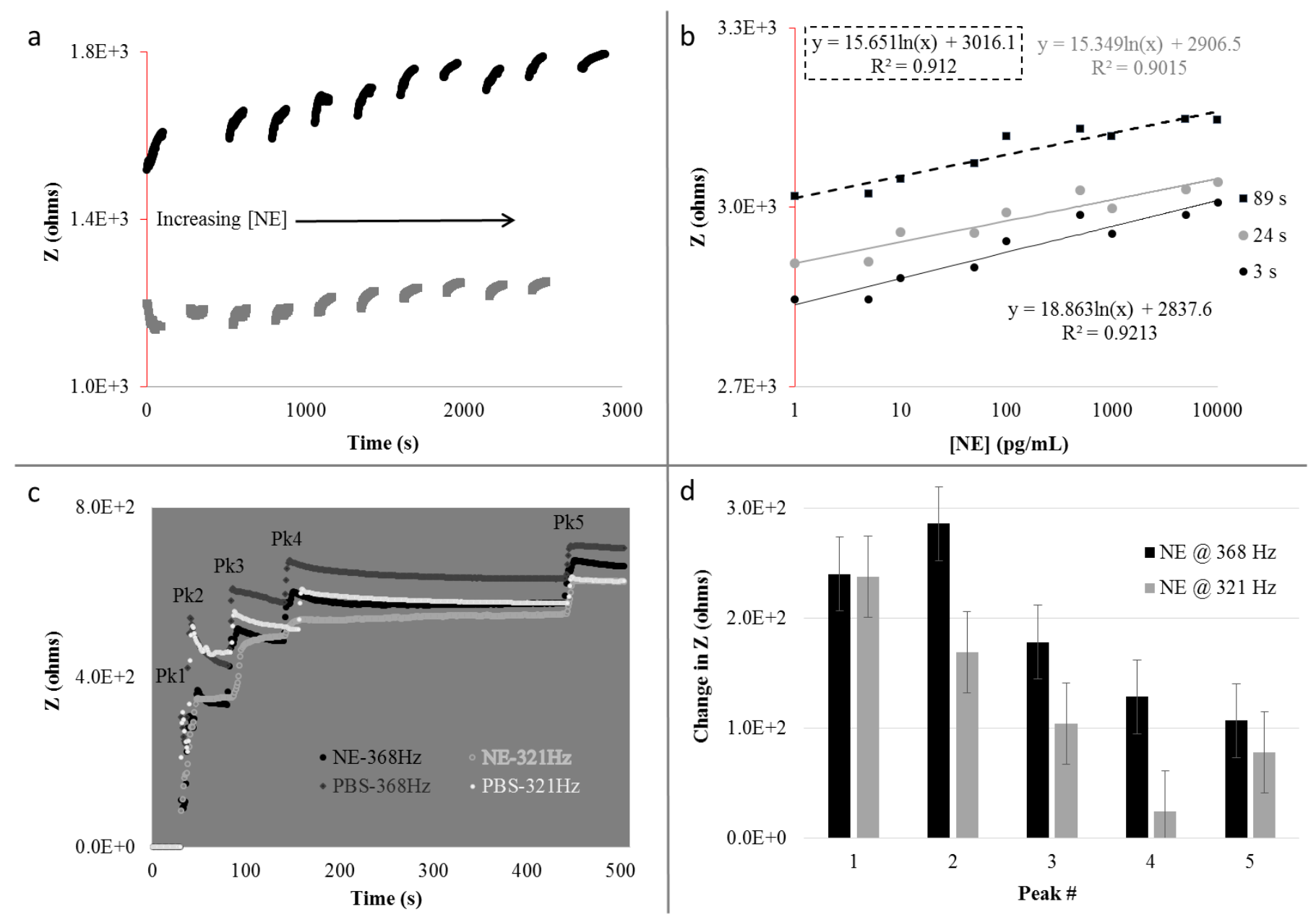

Fig. 2.

(a) An appended plot $(\mathrm{N}=2)$ of the same NE/SAM $0-10,000 \mathrm{pg} / \mathrm{mL}$ gradient used in EIS using instead the Z-t technique at $368 \mathrm{~Hz}$. (b) A calibration curve of complex impedance as a function of NE to determine the optimal time point to collect data. (c) Continuous impedance measurements at two frequencies during spikes of $500 \mathrm{pg} / \mathrm{mL}$ $(2.96 \mathrm{nM}) \mathrm{NE} / \mathrm{SAM}$ or just PBS samples. Each Pk indicates a time at which PBS or $500 \mathrm{pg} / \mathrm{mL}$ NE/SAM was injected into solution. (d) This bar chart shows the $\mathrm{N}=2$ average peak height at each injection time point with the injection artifact subtracted out (using the PBS injection data).

\section{Conclusions}

This work has explored the use of impedance-based electrochemical techniques for use in a future point-of-care technology to diagnose and quantify various severities of TBI. With reproducible LLD's ranging from 8 to $98 \mathrm{pg} / \mathrm{mL}(47.29-579.27 \mathrm{pM})$, we have shown that the impedance-based electrochemical techniques are sensitive enough to detect norepinephrine in purified and complex media at physiologically relevant concentrations. Additionally, we have 
explored the feasibility of using the Z-t technique to reduce hardware requirements without sacrificing sensitivity.

Future work will include efforts to reduce noise in complex media via implementation of a membrane over the sensor surface or encapsulating the enzyme in a mesoporous structure. Other efforts will focus of verifying and optimizing the Z-t results in complex media and expanding the use of this technique for simultaneous TBI biomarker detection.

\section{Acknowledgments}

This work was supported by the School of Biological and Heath Systems Engineering at Arizona State University.

\section{References}

Adamson, T. L., Eusebio, F. A., Cook, C. B., \& LaBelle, J. T. 2012. Analyst 137, 4179.

Atta, N. F., El-Kady, M. F., \& Galal, A. 2010. Anal. Biochem. 400, 78.

BRAIN INJURY FACTS. Retrieved June 6, 2014, from http://www.internationalbrain.org/braininjury-facts/

National Hospital Discharge Survey (NHDS). Centers for Disease Control. 2010.

Chamoun, R.B., Gopinath, S.P., Robertson, C.S. 2009. Europ. Crit. Care Emerg. Med. - Touch Breifings 47.

Clifton, G. L., Ziegler, M. G., \& Grossman, R. G. 1981. Neurosurgery 8, 10.

Daniel, J., Ng, T. N., Garner, S., Arias, A. C., Coleman, J., Liu, J., \& Jackson, R. 2010. In Sensors, 2010 IEEE, 2259.

Fairchild, A. B., McAferty, K., Demirok, U. K., \& La Belle, J. T. 2009. CME. ICME International Conference, IEEE, 1.

Faul, M., Xu, L., Wald, M. M., \& Coronado, V. G. 2010. Centers for Disease Control and Prevention, National Center for Injury Prevention and Control, 2.

Finkelstein, E. A., Corso, P. S., \& Miller, T. R. 2006. Oxford University Press.

Ghindilis, A.L., Smith, M. W., Schwarzkopf, K. R., Zhan, C., Evans, D. R., Baptista, A. M., and Simon, H. M. 2009. Electroanalysis 21.13, 1459. 
Gonzales, S.I., and J.T. La Belle. 2012. Biosens. J. 1, 1.

Greenwald, R. M., Gwin, J. T., Chu, J. J., \& Crisco, J. J. 2008. Neurosurgery 62, 789.

Hergenroeder, G. W., Redell, J. B., Moore, A. N., \& Dash, P. K. 2008. Molec. Diagn. Ther. 12, 345 .

Hamill, R. W., Woolf, P. D., McDonald, J. V., Lee, L. A., \& Kelly, M. 1987. Annals of Neuro. 21,438 .

Kim, Y. R., Bong, S., Kang, Y. J., Yang, Y., Mahajan, R. K., Kim, J. S., \& Kim, H. 2010. Biosens. Bioelectron. 25, 2366.

Kumar, A., Hart, J. P., \& McCalley, D. V. 2011. J. Chroma. A 1218, 3854.

La Belle, J. T., Bhavsar, K., Fairchild, A., Das, A., Sweeney, J., Alford, T. L., ... \& Joshi, L. 2007. Biosens. Bioelectron. 23, 428.

La Belle, J. T., Fairchild, A., Demirok, U. K., \& Verma, A. 2013. Methods 61, 39.

Mautes, A. E. M., Müller, M., Cortbus, F., Schwerdtfeger, K., Maier, B., Holanda, M., ... \& Steudel, W. I. 2001. Acta Neurochirurgica, 143(1), 51-58.

Mayo Clinic Laboratories. Test ID: CATP Catecholamine Fractionation, Plasma, Free. CATP. Retrieved June 6, 2014, from http://www.mayomedicallaboratories.com/testcatalog/Clinical+and+Interpretive/8532

National Center for Injury Prevention and Control (US). 2003. Centers for Disease Control and Prevention.

Prasad, B. B., Srivastava, S., Tiwari, K., \& Sharma, P. S. 2009. Biochem. Eng. J. 44, 232.

Raggi, M. A., Sabbioni, C., Casamenti, G., Gerra, G., Calonghi, N., \& Masotti, L. 1999. J. Chroma. B: Biomed. Sci. App. 730, 201.

Salim, A., Hadjizacharia, P., Dubose, J., Brown, C., Inaba, K., Chan, L. S., \& Margulies, D. 2009. The Amer. Surg. 75, 25. 
Svetlov, S. I., Prima, V., Kirk, D. R., Gutierrez, H., Curley, K. C., Hayes, R. L., \& Wang, K. K. 2010. J. Trauma Acute Care Surg. 69, 795.

Tsunoda, M., Yamagishi, M., Imai, K., \& Yanagisawa, T. 2009. Anal. Bioanal. Chem. 394, 947.

Upadhyay, P. and S. Bhaskar. 2000. J. Immun. Meth. 244.1, 133. 